\title{
Urinary microRNA-30a-5p is a potential biomarker for ovarian serous adenocarcinoma
}

\author{
JUN ZHOU ${ }^{1,2}$, GUANGHUI GONG $^{1,2}$, HONG TAN $^{1,2}$, FURONG DAI $^{3}$, XIN ZHU $^{3}$, YILE CHEN $^{4}$, \\ JUNPU WANG $^{1,2}$, YING LIU ${ }^{1,2}$, PUXIANG CHEN ${ }^{5}$, XIAOYING WU $^{1,2^{*}}$ and JIFANG WEN ${ }^{1,2^{*}}$ \\ ${ }^{1}$ Department of Pathology, School of Basic Medical Science, Central South University, Changsha, Hunan 410013; \\ ${ }^{2}$ Department of Pathology, Xiangya Hospital, Central South University, Changsha, Hunan 410008; \\ ${ }^{3}$ Department of Gynecology and Obstetrics, Xiangya Hospital, Central South University, Changsha, Hunan 410008; \\ ${ }^{4}$ Department of Gynecology Oncology, Hunan Tumor Hospital, Changsha, Hunan 410013; \\ ${ }^{5}$ Department of Gynecology and Obstetrics, The Second Xiangya Hospital, \\ Central South University, Changsha, Hunan 410011, P.R. China
}

Received December 14, 2014; Accepted February 13, 2015

DOI: 10.3892/or.2015.3937

\begin{abstract}
MicroRNAs (miRNAs) can serve as biomarkers in human cancer. To determine the clinical value of urinary miRNAs for ovarian serous adenocarcinoma, we collected urine samples from 39 ovarian serous adenocarcinoma patients, 26 patients with benign gynecological disease and 30 healthy controls. The miRNA microarray data showed that only miR-30a-5p was upregulated and 37 miRNAs were downregulated in the urine samples of ovarian serous adenocarcinoma patients, when compared to healthy controls, which was confirmed after conducting quantitative PCR. The upregulation of urinary miR-30a-5p was closely associated with early stage of ovarian serous adenocarcinoma as well as lymphatic metastasis. Receiver operator characteristic (ROC) analysis demonstrated the potential use of urinary miR-30a-5p as a diagnostic marker for ovarian serous adenocarcinoma. Furthermore, a lower urine level of miR-30a-5p was found in 20 gastric cancer and 20 colon carcinoma patients when compared to ovarian serous adenocarcinoma, suggesting that the upregulation of urinary miR-30a-5p may be specific for ovarian serous adenocarcinoma. miR-30a-5p was also upregulated in ovarian serous adenocarcinoma tissues and cell lines, while urinary miR-30a-5p from ovarian cancer patients was notably reduced following the surgical removal of ovarian serous adenocarcinoma, suggesting that urinary
\end{abstract}

Correspondence to: Professor Xiaoying Wu or Professor Jifang Wen, Department of Pathology, School of Basic Medical Science, Central South University, 138 Tongzipo Road, Changsha, Hunan 410013, P.R. China

E-mail: csuwuxiaoying@163.com

E-mail: jifangw@yahoo.com

"Contributed equally

Key words: ovarian serous adenocarcinoma, microRNA, urine, biomarker, non-invasive
miR-30a-5p was derived from the ovarian serous adenocarcinoma tissue. Notably, miR-30a-5p was concentrated with exosomes from the ovarian cancer cell supernatant or urine from ovarian serous adenocarcinoma patients, supporting a pathway for excretion into the urine. The results also showed that the knockdown of miR-30a-5p significantly inhibited the proliferation and migration of ovarian cancer cells. In summary, to the best of our knowledge, the present study provided the first evidence of increased miR-30a-5p in the urine of ovarian serous adenocarcinoma patients, while the inhibition of miR-30a-5p suppressed the malignant phenotypes of ovarian cancer in vitro. Therefore, miR-30a-5p serves as a promising diagnostic and therapeutic target for ovarian serous adenocarcinoma.

\section{Introduction}

Epithelial ovarian cancer remains the fifth most common cancer in women worldwide and the leading cause of mortality for female reproductive system tumors (1). The early symptom of ovarian cancer is obscure and reliable early diagnosis for ovarian cancer is lacking. Although the survival rates of advanced cancer patients have been improved over the past decades, such improvements in ovarian cancer have only been observed in the 5-year survival rate for women with stage III and IV tumors who are $<30$ years old $(1,2)$. Therefore, developing reliable biomarkers for the early diagnosis for ovarian cancer is crucial.

Common diagnostic methods for ovarian cancer include transvaginal ultrasonography, bimanual rectovaginal pelvic examination and the serum level of CA125, an ovarian-related antigenic determinant. However, routine gynecologic examinations lack specificity and sensitivity $(3,4)$. Moreover, although the CA125 serum level is elevated in $80 \%$ of patients with epithelial ovarian cancer, the use of this marker for the early detection of ovarian cancer is limited, since only $50 \%$ of patients with stage I ovarian cancer show elevated CA125 levels. In addition, the CA125 serum level is also increased in 
Table I. Summary description of study subjects.

\begin{tabular}{lcccc}
\hline Variables & $\begin{array}{c}\text { Urine } \\
\text { miRNA microarray } \\
(\mathrm{n})\end{array}$ & $\begin{array}{c}\text { Urine RT-qPCR } \\
(\mathrm{n})\end{array}$ & $\begin{array}{c}\text { Tissue RT-qPCR } \\
(\mathrm{n})\end{array}$ & $\begin{array}{c}\text { Urine total } \\
(\mathrm{n})\end{array}$ \\
\hline Healthy woman & 5 & 25 & 20 & 30 \\
Benign gynecologic disease & 0 & $26^{\mathrm{a}}$ & $21^{\mathrm{d}}$ & $26^{\mathrm{a}}$ \\
Ovarian serous adenocarcinoma & $5^{\mathrm{f}}$ & $34^{\mathrm{e}}$ & $30^{\mathrm{e}}$ & $39^{\mathrm{b}}$ \\
Gastric cancer & 0 & $20^{\mathrm{c}}$ & 0 & $20^{\mathrm{c}}$ \\
Colon carcinoma & 0 & $20^{\mathrm{c}}$ & 0 & $20^{\mathrm{c}}$ \\
\hline
\end{tabular}

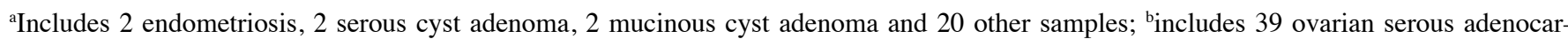

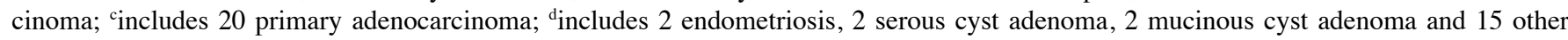
samples; includes 34 ovarian serous adenocarcinoma; fincludes 5 ovarian serous adenocarcinoma.

a significant number of women with benign ovarian lesions as well as in healthy women, which limits its specificity as a diagnostic biomarker (5-7). As yet, no useful biomarker has been identified for ovarian cancer in urine, probably since the majority of efforts have focused on blood protein biomarkers, which are hardly detected in the urine.

MicroRNAs (miRNAs) are small (18-24 nucleotides), highly conserved, non-coding RNAs that regulate gene expression post-transcriptionally (8). Computational studies suggest that $>60 \%$ of mammalian gene transcripts are regulated by miRNAs (9). miRNAs play a key regulatory role in divergent cell processes, such as proliferation, differentiation, apoptosis, migration, as well as tumorigenesis $(10,11)$. miRNAs are aberrantly expressed in human cancer, and the miRNA profiling of poorly differentiated tumors was more accurate than the mRNA-profiling, suggesting a potential clinical value of miRNAs in the diagnosis of cancer (12).

On a technical level, urine samples are easier to obtain and contain fewer proteins than blood-based samples, reducing the interference of proteins during RNA extraction and the subsequent analysis. In addition to miRNAs that may be excreted locally by the urinary system, urine miRNAs may be derived from the circulating blood via renal elimination $(13,14)$. A number of miRNAs have been identified in the urine (15-17), suggesting the potential utility of urine miRNAs for identifying or qualifying ovarian cancer.

In the present study, we aimed to examine the roles of urine miRNAs in the diagnosis of ovarian serous adenocarcinoma. In addition, we preliminarily explored the underlying mechanisms involved.

\section{Materials and methods}

Ethics statement. The present study was approved by the Clinical Research Ethics Committee of Central South University, and the EC (S) ID no. 201406374. In addition, the patients involved in the present study provided written informed consent.

Urine and tissue samples. Morning whole-stream urine samples were collected at the time of initial consultation prior to therapy at the Xiangya Hospital, Central South University (Changsha, China). As shown in Table I, urine samples were collected from 39 ovarian serous adenocarcinoma patients, 26 patients with benign gynecological disease and 30 age-matched healthy women used as normal controls. Women with benign gynecologic diseases had a variety of pathological diagnoses, including benign ovarian cyst adenoma, uterine fibroids, endometriosis and physiological ovarian cysts (Table I). Urine samples from 20 gastric cancer and 20 colon carcinoma patients were also collected (Table I). Moreover, a total of 51 tissue specimens were collected, including 21 benign gynecologic diseases specimens and 30 ovarian serous adenocarcinoma tissues (Table I).

Cell lines. The SKOV-3, SKOV-3ip, HO-8910, HO-8910pm human ovarian carcinoma cell lines and the 293-T and U-937 non-ovarian cell lines were obtained from the American Type Culture Collection (ATCC; Manassas, VA, USA). The normal OSE ovarian surface epithelium cell lines was used as a non-cancerous control in the present study. The cell lines were maintained at $37^{\circ} \mathrm{C}$ in a humidified atmosphere of $5 \% \mathrm{CO}_{2}$. OSE, SKOV-3, SKOV-3ip and HO-8910 were cultured in RPMI-1640 supplemented with $10 \%$ fetal bovine serum (FBS) (both from Life Technologies, Carlsbad, CA, USA). HO-8910pm, 293-T and U-937 cell lines were cultured in Dulbecco's modified Eagle's medium (DMEM; Life Technologies) with 10\% FBS.

Preparation of RNA. The mirVana miRNA Isolation kit (Ambion, Austin, TX, USA) was used for the extraction of total RNA from the urine sample cell supernatants and urine exosomes according to the manufacturer's instructions. For each subject, $600 \mu 1$ of urine was used for miRNA extraction. Total RNA content was extracted from tissues and cells using TRIzol reagent (Ambion).

miRNA microarray analysis. miRNA microarray was performed at the Beijing CapitalBio Corporation. miRNA (200 ng) isolated from each urine sample was labeled with cyanine 3-pCp using Agilent miRNA labeling and hybridization kits, hybridized to the Agilent Human miRNA Microarray (miRBase release 19.0), and then scanned. The fluorescence intensities were converted into digital data and $\log 2$ transformed using Feature Extraction (version 10.7). Genes that were differentially expressed between ovarian cancer patients and healthy controls were analyzed using GeneSpring software version 12.0 (Agilent). miRNAs that fulfilled the criteria of a 
P-value $(\%)<0.05$ and a fold-change $\geq 2$ between the groups were considered to be significantly different. Heat maps were generated using the Cluster 3.0 package software. Our data was uploaded in the Gene Expression Omnibus (GEO) of the National Center for Biotechnology Information (NCBI) (accession no. GSE58517).

Supervised clustering analysis of microarray miRNAs. Prior to hierarchical clustering, miRNA profiles were standardized to a mean of zero and a standard deviation of 1.0. The clustering analysis was performed with average linkage and the Pearson's correlation. Briefly, the purpose of this algorithm is to assemble all elements into a single tree by calculating a dendrogram. For any set of miRNA genes, an upper-diagonal similarity matrix was computed. All the pairs of genes were assigned similarity scores. The highest value was scanned to identify the matrix (representing the most similar miRNAs). The paired genes formed a node that was used to compute a gene expression profile for the joined elements by averaging observation: after the missing values were omitted, the gene expression of the two joined elements was determined by the number of miRNAs they contained.

Reverse transcription-quantitative real-time polymerase chain reaction. The TaqMan microRNA reverse transcription kit (Applied Biosystems, Foster City, CA, USA) and High Capacity cDNA Reverse Transcription kit (Applied Biosystems) were used for reverse transcription. For miRNA amplification, $5 \mu \mathrm{l}$ total RNA was mixed with $0.15 \mu \mathrm{l}$ of $100 \mathrm{~mm}$ dNTPs (with dTTP), $1 \mu \mathrm{l}$ (50 units) MultiScribe reverse transcriptase, $1.5 \mu \mathrm{l}$ of $10 \mathrm{X}$ reverse transcription buffer, $0.19 \mu \mathrm{l}$ RNase inhibitor $(20 \mathrm{U} /$ $\mu \mathrm{l}$ ), and $3 \mu \mathrm{l} 1 \mathrm{X}$ gene-specific primers. Nuclease-free $\mathrm{H}_{2} \mathrm{O}$ was added to obtain a final volume of $15 \mu \mathrm{l}$. Reverse transcription was performed at $16^{\circ} \mathrm{C}$ for $30 \mathrm{~min}$, followed by an incubation step at $42^{\circ} \mathrm{C}$ for $30 \mathrm{~min}$ and enzyme inactivation at $85^{\circ} \mathrm{C}$ for $5 \mathrm{~min}$. The resulting cDNA was stored at $-20^{\circ} \mathrm{C}$ until use. The cDNA was quantified by reverse transcription-quantitative polymerase chain reaction (RT-qPCR) using the ABI PRISM 7500 Sequence Detection System (Applied Biosystems). For RT-qPCR, $0.33 \mu \mathrm{l}$ cDNA solution, $10 \mu \mathrm{l}$ of $1 \mathrm{X}$ TaqMan universal PCR master mix, $2 \mu \mathrm{l}$ of $1 \mathrm{X}$ gene specific primer/probe set (all from Applied Biosystems) and $7.67 \mu \mathrm{l}$ RNase-free $\mathrm{H}_{2} \mathrm{O}$ were mixed to obtain a final reaction volume of $20 \mu \mathrm{l}$. Each sample was run in triplicate. The RT-qPCR steps were $50^{\circ} \mathrm{C}$ for $2 \mathrm{~min}, 95^{\circ} \mathrm{C}$ for $10 \mathrm{~min}$ and 40 cycles of denaturation at $95^{\circ} \mathrm{C}$ for $15 \mathrm{sec}$, followed by an annealing/elongation step at $60^{\circ} \mathrm{C}$ for $60 \mathrm{sec}$. The miR30a-5p and miR-6076 were detected in triplicate via RT-qPCR, and RNU6 (Applied Biosystems) was used as the housekeeping gene to normalize the miRNA expression in the urine. The data were analyzed with the SDS relative quantification software version 2.2.2 (Applied Biosystems). The same baseline and cycle threshold (CT) were set for each target. The $2^{-\Delta \Delta \mathrm{Ct}}$ method was used for relative quantization of the differences of the expression levels of each target in the urine samples. The absolute amount of cell supernatant and urine exosome miR-30a-5p was calculated by software based on the sample RT-qPCR numbers and the standard curve, and were expressed as pmol/l.

Exosome isolation from the cell supernatant or urine. Exosomes from the cell supernatant and urine were isolated by ultracentrifugation. Briefly, the urine was centrifuged at $2,000 \times \mathrm{g}$ for $20 \mathrm{~min}$ to eliminate the cells and debris and then at $13,500 \times \mathrm{g}$ for $20 \mathrm{~min}$, followed by ultracentrifugation (Beckman L-80XP 70Ti) at 200,000 x g for $1 \mathrm{~h}$ to pellet the exosomes. The exosome pellets were resuspended in phosphate-buffered saline (PBS) prior to experimentation.

Electron microscopy of exosomes. Exosomes were isolated from the cell supernatant and urine. The exosomes were washed in PBS, filtered and ultracentrifugation was performed at $200,000 \mathrm{x}$ g for $1 \mathrm{~h}$ to re-pellet the exosomes. The exosome pellet was resuspended and fixed in PBS containing 2\% glutaraldehyde and then loaded onto formvar/carbon-coated electron microscopy grids. The samples were contrasted with uranyl acetate to visualize the membrane and viewed with a FEI Tecnai G2 Spirit electron microscope.

Transfection. Lipofectamine 2000 (Life Technologies) was used to perform cell transfection following the manufacturer's instructions. Briefly, ovarian cancer cells were transfected with scramble miRNA (NC) or miR-30a-5p inhibitor (Nlunbio Co., Changsha, China).

Cell proliferation assay. The MTT assay was used to measure the cell proliferation of ovarian cancer cells transfected with scramble miRNA (NC) or miR-30a-5p inhibitor. At $48 \mathrm{~h}$ post-transfection, the transfection medium in each well was replaced with $100 \mu \mathrm{l}$ fresh serum-free medium containing $0.5 \mathrm{~g} / \mathrm{l} \mathrm{MTT}$. Subsequent to incubation at $37^{\circ} \mathrm{C}$ for $4 \mathrm{~h}$, the MTT medium was removed by aspiration and $50 \mu 1$ dimethylsulfoxide was added to each well. Following incubation at $37^{\circ} \mathrm{C}$ for an additional $10 \mathrm{~min}$, the optical density at $570 \mathrm{~nm}$ was measured using the Bio-Tek ${ }^{\mathrm{TM}} \mathrm{ELx} 00^{\mathrm{TM}}$ absorbance microplate reader (Bio-Tek, Winooski, VT, USA). This experiment was repeated three times.

Cell migration assay. A wound-healing assay was performed to evaluate the cell migratory capacity of ovarian cancer cells transfected with scramble miR (NC) or miR-30a-5p inhibitor. Briefly, the cells were cultured to full confluence. Wounds of $\sim 1 \mathrm{~mm}$ width were created with a plastic scriber, and the cells were washed and incubated in serum-free medium. After wounding for $24 \mathrm{~h}$, the cells were incubated in a medium including $10 \%$ FBS. After further culturing for 0 and $48 \mathrm{~h}$, the cells were fixed and observed under a microscope.

Statistical analysis. Values are presented as the mean \pm SD. Statistical analysis was performed using SPSS 16.0 (SPSS, Inc., IBM, Armonk, NY, USA). An unpaired Student's t-test was used to compare the miRNA expression in different groups. $\mathrm{P}<0.05$ indicated significant differences.

\section{Results}

Microarray identification of differentially expressed miRNAs in urine samples from patients with ovarian serous adenocarcinoma. To identify differentially expressed miRNAs in urine from ovarian serous adenocarcinoma patients, urine samples were collected from 5 ovarian serous adenocarcinoma patients and 5 age-matched healthy female control subjects. miRNA 


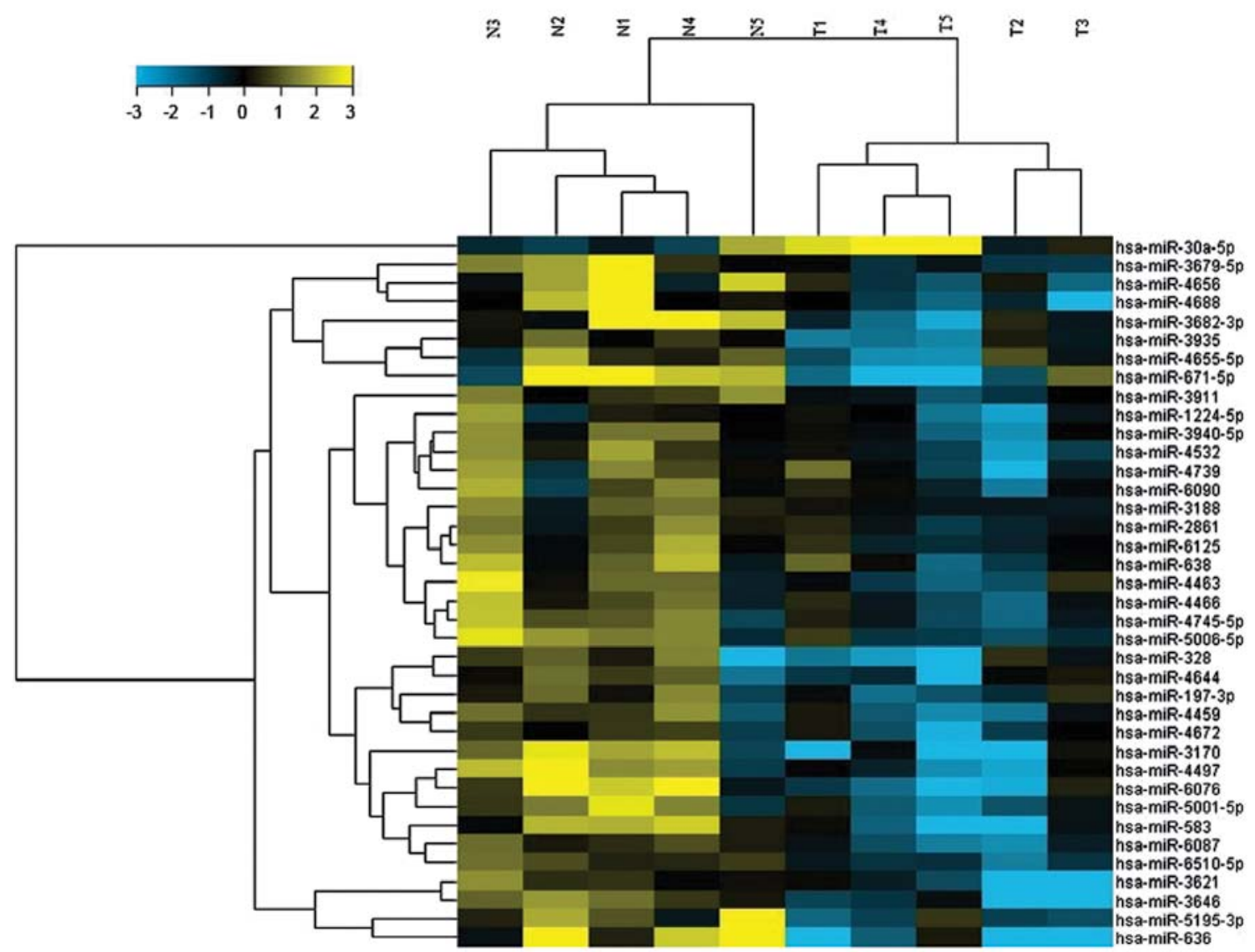

Figure 1. Supervised hierarchical clustering of differential miRNA expression profiles for ovarian serous adenocarcinoma. Microarray analysis for miRNAs was performed in the urine from 5 ovarian serous adenocarcinoma patients and 5 healthy controls. The heat map diagram shows that the expression of one miRNA (miR-30a-5p) was upregulated while 37 miRNAs were downregulated $>2$-fold with $\mathrm{P}<0.05$. Each row represents an miRNA, and each column a sample. The color scale shown at the top shows the relative miRNA expression. Yellow shows a high expression and blue a low expression. N1-N5 are RNA samples from healthy women. T1-T5 are RNA samples from ovarian serous adenocarcinoma patients. The blocks corresponding to the two genes that were further assessed in the present study (miR-30a-5p and miR-6076) are indicated.

expression profiling was conducted using the Agilent Human miRNA Microarray (miRBase release 19.0), which contains probes for 2,006 human miRNAs. miRNAs with a mean change $>2.0$-fold and $\mathrm{P}<0.05$ between ovarian cancer and healthy control samples were considered significantly different. As shown in Fig. 1 and Table II, one miRNA was upregulated and 37 miRNAs were downregulated in the ovarian serous adenocarcinoma samples compared to the healthy controls. To validate the microarray analysis, we compared the relative level of representative miRNAs in the urine samples from an independent set of 34 ovarian serous adenocarcinoma patients, 26 patients with benign gynecological disease and 25 healthy controls. The upregulated miRNA identified by microarray analysis (miR-30a-5p) and a representative downregulated miRNA (miR-6076) were assessed. As shown in Fig. 2A, the relative miR-30a-5p level in ovarian serous adenocarcinoma specimens was 4.6 -fold higher when compared with the healthy controls $(\mathrm{P}<0.05)$, and 9.0-fold higher when compared with the benign ovarian specimens $(\mathrm{P}<0.05)$. Additionally, the relative miR-30a-5p level in the benign ovarian specimens was lower than that in the healthy controls, although this difference was not statistically significant ( $\mathrm{P}>0.05$; Fig. 2A). Moreover, the results suggested that the upregulation of urinary miR$30 a-5 p$ was closely associated with the early stage of ovarian serous adenocarcinoma, since its expression level was higher in stage I-II ovarian serous adenocarcinoma samples than that in stage III-IV samples $(\mathrm{P}<0.01)$, and higher in well or well-moderately-differentiated ovarian serous adenocarcinoma samples than in poorly-differentiated samples $(\mathrm{P}<0.05$, Table III). The results suggested that the upregulation of urinary miR-30a-5p was associated with lymphatic metastasis $(\mathrm{P}<0.01$, Table III).

Furthermore, the relative miR-6076 level was significantly reduced ( $>10$-fold reduction) in the urine of ovarian serous adenocarcinoma patients, when compared to the healthy controls and the patients with benign gynecological disease, respectively $(\mathrm{P}<0.05$; Fig. $2 \mathrm{~B})$, These findings are consistent with the miRNA microarray data, and suggest that an increased miR-30a-5p and a decreased miR-6076 may serve as promising biomarkers that distinguish ovarian serous adenocarcinoma from other gynecological diseases.

Assessment of the diagnostic value of miR-30a-5p and $m i R-6076$ for ovarian serous adenocarcinoma. To assess the diagnostic potential of urinary miR-30a-5p and miR-6076 levels for ovarian serous adenocarcinoma, we performed a receiver operator characteristic (ROC) curve analysis of specificity and sensitivity of the 34 ovarian serous 
Table II. Differently expressed miRNAS in the urine of ovarian serous adenocarcinoma patients vs. healthy control subjects.

\begin{tabular}{|c|c|c|}
\hline miRNA & P-value & Fold-change \\
\hline \multicolumn{3}{|l|}{ Upregulated } \\
\hline hsa-miRNA-30a-5p & 0.02159635 & 4.668867 \\
\hline \multicolumn{3}{|l|}{ Downregulated } \\
\hline hsa-miR-636 & 0.0202967 & 64.08348 \\
\hline hsa-miR-3170 & 0.0248452 & 52.002758 \\
\hline hsa-miR-671-5p & 0.0176856 & 20.946095 \\
\hline hsa-miR-583 & 0.0356045 & 14.240913 \\
\hline hsa-miR-6076 & 0.0146871 & 12.56148 \\
\hline hsa-miR-3682-3p & 0.0465591 & 11.362884 \\
\hline hsa-miR-4688 & 0.0284103 & 10.914472 \\
\hline hsa-miR-3646 & 0.0430764 & 10.0874815 \\
\hline hsa-miR-3621 & 0.0498169 & 8.299675 \\
\hline hsa-miR-4497 & 0.0360293 & 6.8510914 \\
\hline hsa-miR-5195-3p & 0.0154927 & 5.6532087 \\
\hline hsa-miR-4656 & 0.029555 & 4.497623 \\
\hline hsa-miR-5001-5p & 0.0007499 & 4.3971524 \\
\hline hsa-miR-3679-5p & 0.0225566 & 4.358498 \\
\hline hsa-miR-5006-5p & 0.0363791 & 3.9775543 \\
\hline hsa-miR-6087 & 0.0063278 & 3.7868533 \\
\hline hsa-miR-4532 & 0.004153 & 3.615911 \\
\hline hsa-miR-6510-5p & 0.0111118 & 3.306896 \\
\hline hsa-miR-4655-5p & 0.0250463 & 3.2763517 \\
\hline hsa-miR-4459 & 0.0215316 & 3.2297115 \\
\hline hsa-miR-328 & 0.0284044 & 3.2181585 \\
\hline hsa-miR-4463 & 0.0395245 & 3.191302 \\
\hline hsa-miR-3935 & 0.002951 & 3.1714096 \\
\hline hsa-miR-3940-5p & 0.0007361 & 3.157188 \\
\hline hsa-miR-4745-5p & 0.0373854 & 3.0417292 \\
\hline hsa-miR-3911 & 0.0342525 & 2.7798533 \\
\hline hsa-miR-4466 & 0.0265112 & 2.7150042 \\
\hline hsa-miR-4739 & 0.0150427 & 2.6702106 \\
\hline hsa-miR-638 & 0.0410605 & 2.4692678 \\
\hline hsa-miR-1224-5p & 0.0308876 & 2.3942 \\
\hline hsa-miR-4672 & 0.0159597 & 2.3273127 \\
\hline hsa-miR-6125 & 0.0495394 & 2.2485054 \\
\hline hsa-miR-4644 & 0.0017442 & 2.2420375 \\
\hline hsa-miR-197-3p & 0.0437008 & 2.1905556 \\
\hline hsa-miR-6090 & 0.0250247 & 2.1693227 \\
\hline hsa-miR-2861 & 0.0368039 & 2.080642 \\
\hline hsa-miR-3188 & 0.0407896 & 2.0413487 \\
\hline
\end{tabular}

adenocarcinoma and 25 healthy control samples. The ROC curve for miR-30a-5p demonstrated high specificity and sensitivity, with an area under the ROC curve (AUC) of 0.862 (95\% confidence interval, 0.709-1.016), which suggests a high discrimination potential (Fig. 2C). However, miR-6076 showed a lower specificity and sensitivity, with an AUC of only 0.693 (95\% confidence interval, 0.482-0.904) (Fig. 2D). These results suggested that miR-30a-5p provides a useful
Table III. Clinicopathological characteristics of ovarian serous adenocarcinoma in urine.

\begin{tabular}{lccc}
\hline & \multicolumn{2}{c}{$\begin{array}{c}\text { Relative } \\
\text { miR-30a-5p } \\
\text { expression }\end{array}$} & P-value \\
\hline Mean age (years) & No. & & \\
$\quad<50$ & 21 & $1.84 \pm 0.41$ & $>0.05$ \\
$\geq 50$ & 13 & $2.24 \pm 0.49$ & \\
Regional lymph node & & & \\
involvement & & $1.73 \pm 0.31$ & $<0.001$ \\
$\quad$ No & 6 & $3.21 \pm 0.91$ & \\
$\quad$ Yes & 28 & & \\
Clinical stage & & $2.68 \pm 0.49$ & $<0.001$ \\
$\begin{array}{l}\text { I-II } \\
\text { III-IV }\end{array}$ & 16 & $1.38 \pm 0.34$ & \\
Histological grade & & & $<0.05$ \\
$\quad$ Well-moderately- & 12 & $2.80 \pm 0.56$ & \\
differentiated & & & \\
Poorly-differentiated & 22 & $1.55 \pm 0.35$ & \\
\hline
\end{tabular}

diagnostic biomarker for ovarian serous adenocarcinoma, in contrast to miR-6076.

Upregulation of urinary miR-30a-5p level may be specific for ovarian cancer. We investigated whether urinary miR-30a-5p is elevated in other types of cancer or is specific for ovarian cancer. The urinary miR-30a-5p level was examined in 34 ovarian serous adenocarcinoma patients, 25 healthy controls, 20 gastric cancer patients and 20 colon carcinoma patients. Whereas the ovarian serous adenocarcinoma samples showed an elevated miR-30a-5p level compared with the healthy controls, the urine samples from the gastric cancer patients and colon carcinoma patients showed a reduced miR-30a-5p level (Fig. 3A). The urinary miR-30a-5p levels of the gastric cancer and colon carcinoma samples were 0.5 - and 0.3 -fold that of the healthy control samples, respectively, although this difference was not statistically significant $(\mathrm{P}>0.05)$ (Fig. 3A). These results suggested that upregulation of the urinary miR-30a-5p level may be specific for ovarian serous adenocarcinoma.

Urinary miR-30a-5p is dervied from the ovarian tumor tissue. To determine whether the urinary miR-30a-5p was derived from ovarian serous adenocarcinoma tissues, we first determined the miR-30a-5p level in tissue samples from 30 ovarian serous adenocarcinoma patients, 21 patients with benign gynecological disease and 20 healthy controls. As shown in Fig. 3B, the relative miR-30a-5p level in ovarian serous adenocarcinoma tissues was 3.2-fold higher than that in the healthy controls $(\mathrm{P}<0.05)$ and 4.0 -fold higher than that in the benign ovarian specimens $(\mathrm{P}<0.05)$. Although the relative miR-30a-5p expression level in the benign ovarian cancer specimens was slightly lower than that in the healthy controls, this difference was not statistically significant (P>0.05) (Fig. 3B). Similar to the expression differences of 
A

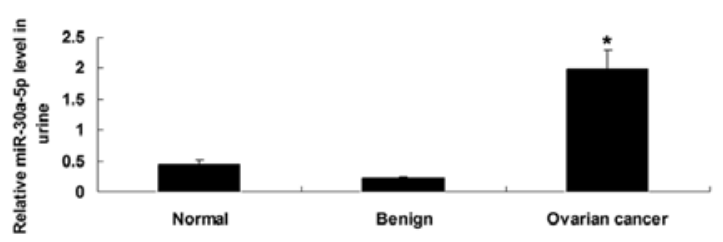

C

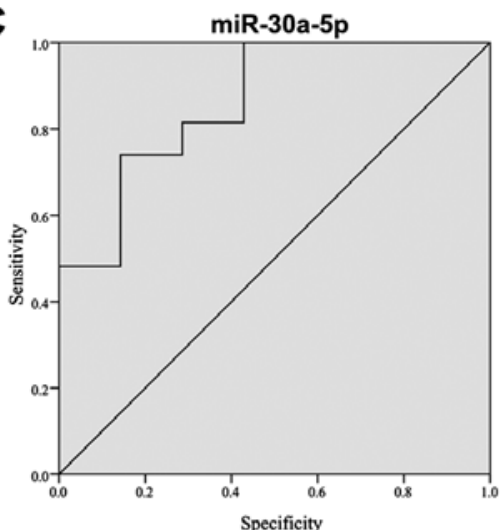

B

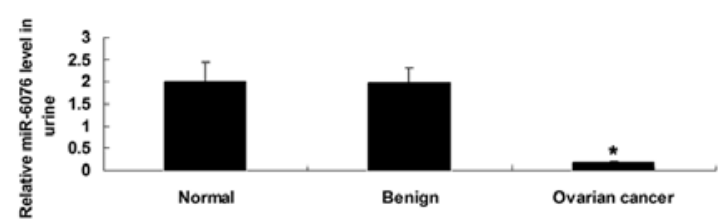

D

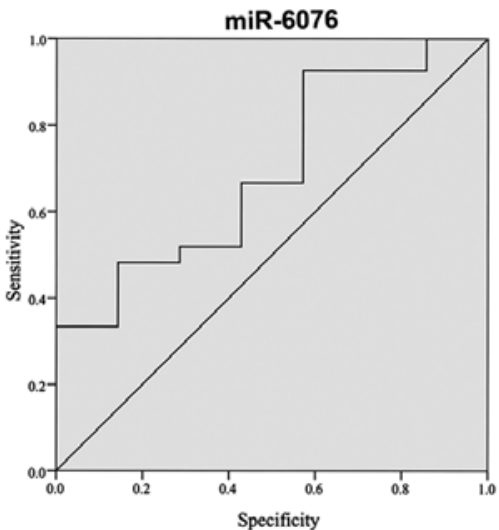

Figure 2. Verification of differential miRNA expression by RT-qPCR. The relative expression levels of (A) miR-30a-5p and (B) miR-6076 in the urine of healthy controls (normal; $n=25$ ), patients with benign gynecological disease (benign; $n=26$ ) and ovarian serous adenocarcinoma patients $(n=34)$ are shown. Data are standardized to RUN6 expression and normalized to 1.0 in the control samples. "P $<0.05$ vs. normal. Receiver operating characteristics (ROC) curve analysis was performed to assess the ability of urinary (C) miR-30a-5p and (D) miR-6076 levels to distinguish the ovarian serous adenocarcinoma cases ( $\mathrm{n}=34$ ) from the control cases $(n=25)$. The areas under the curves (AUCs) for miR-30a-5p and miR-6076 were 0.862 (95\% confidence interval, 0.709-1.016) and 0.693 (95\% confidence interval, 0.482-0.904), respectively.

A
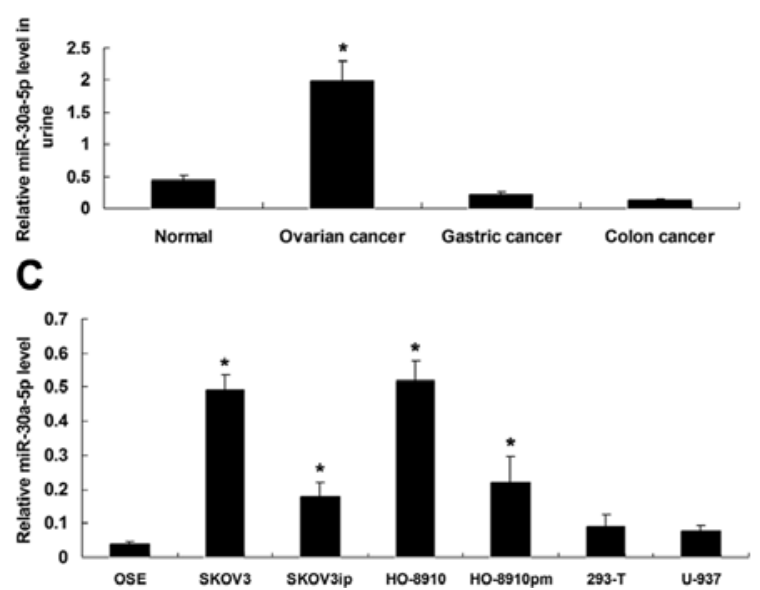

B
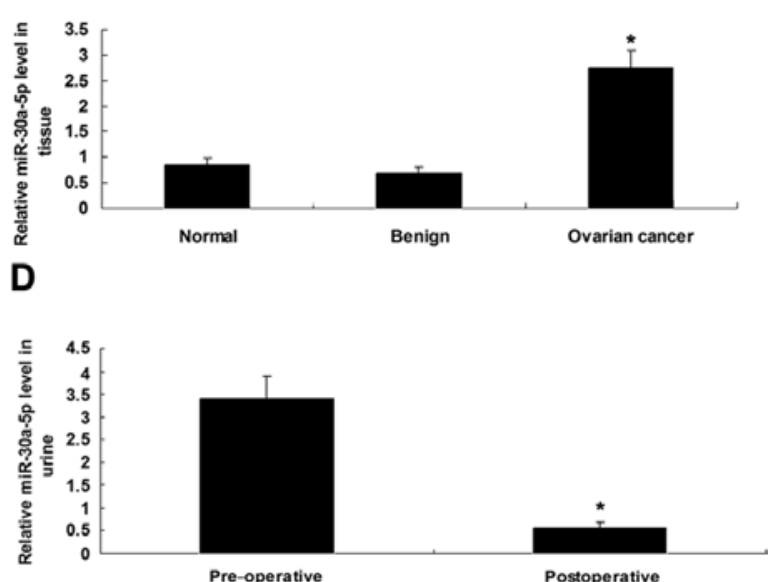

Figure 3. Elevated urinary miR-30a-5p level is specific to ovarian cancer. (A) The level of miR-30a-5p was determined by RT-qPCR in the urine of healthy controls (normal; $n=25)$, gastric cancer patients $(n=20)$, colon carcinoma patients $(n=20)$ and ovarian serous adenocarcinoma patients $(n=34)$. ${ }^{*} \mathrm{P}<0.05$ vs. normal. (B) Relative level of miR-30a-5p in normal ovarian tissues (normal; $n=20$ ), benign gynecological disease tissues (benign; $n=21$ ) and ovarian serous adenocarcinoma tissues ( $\mathrm{n}=30$ ) was determined by RT-qPCR. " $\mathrm{P}<0.05$ vs. normal. (C) Relative level of miR-30a-5p in normal human ovarian surface epithelial (OSE), SKOV-3, SKOV-3ip, HO-8910, HO-8910pm, 293-T and U-937 cells was determined by RT-qPCR. "P<0.05 vs. OSE. (D) The relative level of miR-30a-5p in the urine of 15 ovarian cancer patients was determined by RT-qPCR before and after surgical resection of tumor tissues, respectively. ${ }^{*} \mathrm{P}<0.05$ vs. pre-operative.

miR-30a-5p among the urine samples, these data confirmed that miR-30a-5p was also upregulated in ovarian serous adenocarcinoma tissues. Subsequently, the miR-30a-5p level were determined in normal human ovarian surface epithelial OSE cells, low-invasion human ovarian cancer SKOV-3 and HO-8910 cells, high-invasion human ovarian cancer SKOV-3ip and HO-8910pm cells, human embryonic kidney 293-T cells and human U-937 macrophages. Notably, miR-30a-5p was most highly expressed in all four ovarian cancer cell lines, when compared to other cell types (Fig. 3C), confirming the association of miR-30a-5p upregulation with ovarian cancer.

To confirm that miR-30a-5p in the urine was derived from ovarian cancer cells, we determined whether the urinary miR-30a-5p level was affected by the surgical removal of ovarian serous adenocarcinoma. Assessment of the relative pre-operative and postoperative miR-30a-5p levels for 15 ovarian serous adenocarcinoma patients demonstrated that miR-30a-5p levels were 6-fold higher prior to surgery, than 


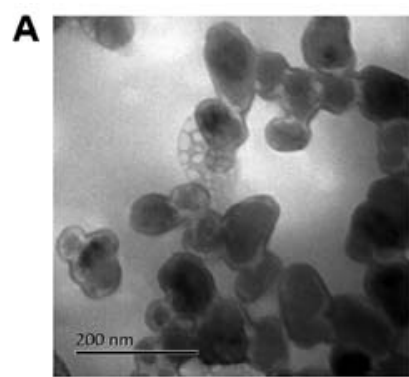

C

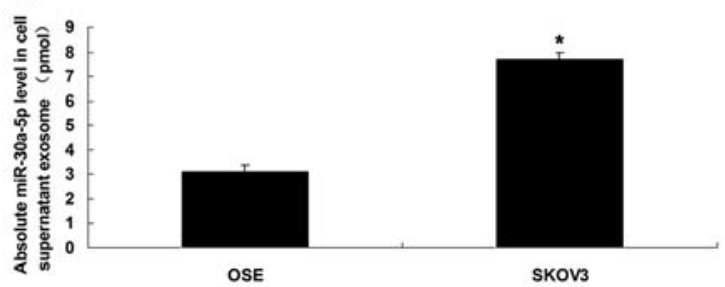

B

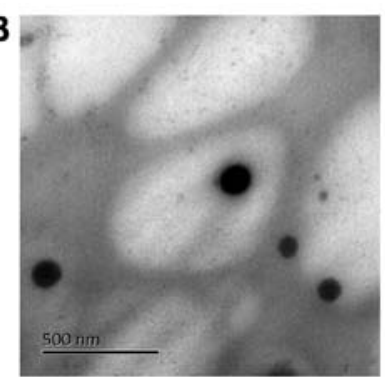

D

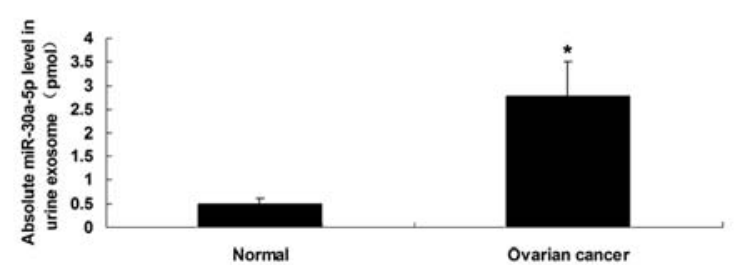

Figure 4. miR-30a-5p is concentrated within the exosomes of cell supernatants and urine. Representative images of (A) cell supernatant and (B) urine exosomes isolated by ultracentrifugation were obtained using an electron microscopy. (C) The level of miR-30a-5p in OSE and SKOV-3 cell supernatant exosomes was determined by RT-qPCR. "P<0.05 vs. OSE. (D) The level of miR-30a-5p in urine exosomes from healthy controls and ovarian serous adenocarcinoma patients was determined by RT-qPCR. ${ }^{*} \mathrm{P}<0.05$ vs. normal.

A

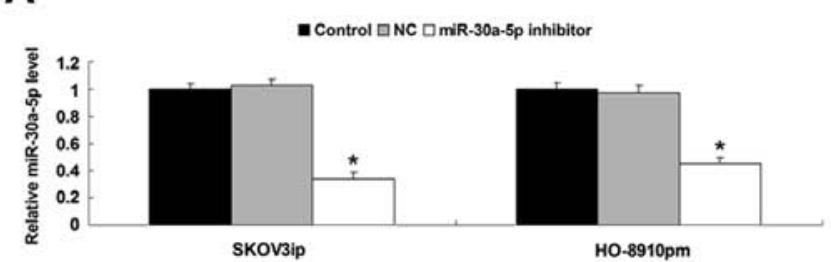

B

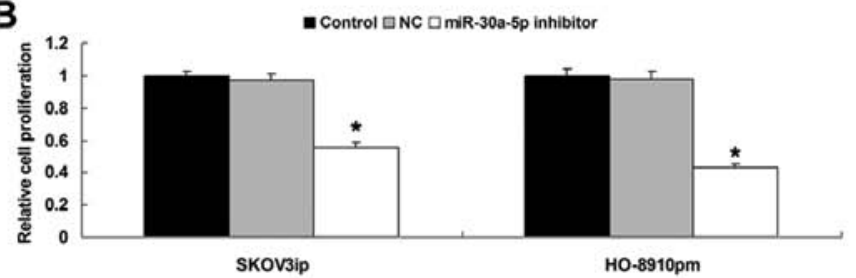

C
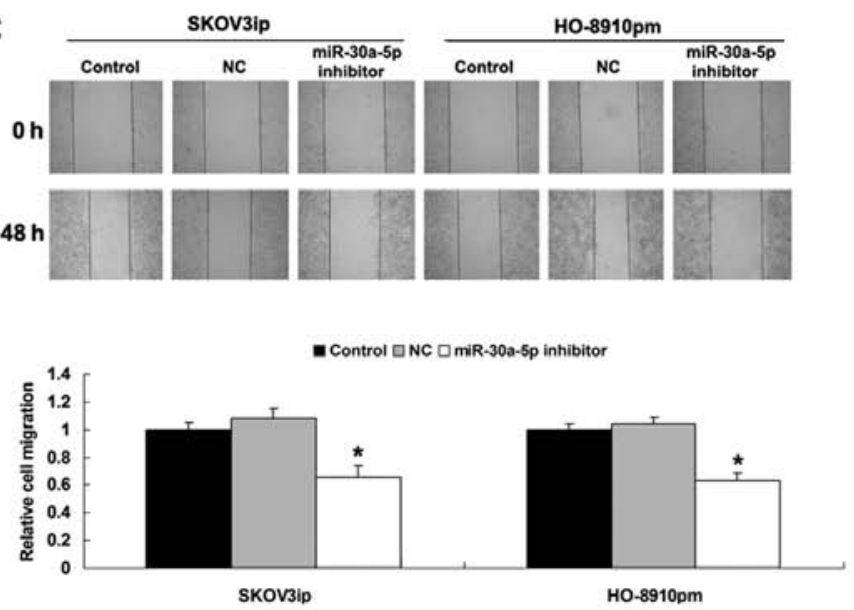

Figure 5. Knockdown of miR-30a-5p significantly inhibits cell proliferation and migration of ovarian cancer cells. (A) RT-qPCR was conducted to detect the expression level of miR-30a-5p in SKOV-3ip and HO-8910pm cells transfected with scramble miR (NC) or miR-30a-5p inhibitor, respectively. (B) Cell proliferation and (C) migration were determined in SKOV-3ip and HO-8910pm cells transfected with scrambled miR (NC) or miR-30a-5p inhibitor, respectively. Control, cells without any transfection. ${ }^{*} \mathrm{P}<0.05$ vs. control.

after surgery (Fig. 3D). The reduced urine level of miR-30a-5p after surgery provided evidence that the urinary miR-30a-5p level is associated with the presence of ovarian serous adenocarcinoma, further supporting that the urinary miR-30a-5p is derived from ovarian serous adenocarcinoma tissues.

Urinary miR-30a-5p is derived from the exosomes secreted by ovarian cancer cells. Aberrant activity of cellular export machinery contributes to cancer development. To determine whether the urinary miR-30a-5p was derived from exosomes, which are cell-derived vesicles that are present in urine and other body fluids, we compared the miR-30a-5p levels in exosomes isolated from the supernatant of SKOV-3 (Fig. 4A) vs. OSE cells, and urine samples (Fig. 4B) from 6 ovarian serous adenocarcinoma patients vs. 6 healthy controls. The miR-30a-5p level in SKOV-3 exosomes was 2.5-fold higher than that in OSE exosomes ( $\mathrm{P}<0.05$; Fig. 4C). Furthermore, the miR-30a-5p level in the urine exosomes from ovarian serous adenocarcinoma patients was 3.3 -fold higher than that from the healthy controls (Fig. 4D). The results suggested that the increased miR-30a-5p level in the urine of ovarian cancer patients may be due to the secretion of exosomes from the ovarian cancer cells.

Knockdown of miR-30a-5p significantly inhibits cell proliferation and migration of ovarian cancer cells. We preliminarily investigated the role of miR-30a-5p in the regulation of malignant phenotypes of ovarian cancer in vitro. We 
transfected SKOV-3ip and HO-8910pm cells with scrambled miRNA or miR-30a-5p inhibitor, respectively. After transfection, RT-qPCR was conducted to detect the expression level of miR-30a-5p in each group. As shown in Fig. 5A, the expression level of miR-30a-5p was notably downregulated in ovarian cancer cells transfected with miR-30a-5p inhibitor compared to the control group $(\mathrm{P}<0.05)$. However, transfection with scrambled miRNA did not affect the miR-30a-5p level in ovarian cancer cells. These results suggested that our transfection was successful. Subsequently, we determined the proliferation and migration abilities of ovarian cancer cells in each group. As shown in Fig. 5B and C, knockdown of miR-30a-5p significantly suppressed the cell proliferation and migration of ovarian cancer cells, when compared to the control group $(\mathrm{P}<0.05)$. These results suggested that miR$30 \mathrm{a}-5 \mathrm{p}$ acts as an oncogenic miRNA in ovarian cancer.

\section{Discussion}

MicroRNAs (miRNAs) can be detected in blood, urine, breast milk, tears, colostrum, bronchial lavage, seminal and pleural fluids. The diagnostic and prognostic value of miRNAs in the urine has been suggested for a variety of diseases $(16,17)$. Previous findings have shown that the different expression patterns of miRNAs in tissues can be used to distinguish ovarian cancers from normal tissue (18). However, the miRNA profiling from tissues is only available to patients undergoing surgery. Accumulating evidence has demonstrated the imbalanced production of some miRNAs in the circulation $(19,20)$. However, blood tests was invasive, limiting its clinical utility. Collection of urine is more convenient, cost-effective and non-invasive, when compared to serum, plasma and tissue specimens. Therefore, we examined whether miRNAs in the urine served as non-invasive biomarkers for ovarian cancer.

Our miRNA microarray results showed that 38 miRNAs were significantly differentially expressed in the urine from ovarian serous adenocarcinoma patients compared to healthy controls, including one (miR-30a-5p) upregulated miRNA and 37 downregulated miRNAs. Further investigation found that the urinary miR-30a-5p in gastric cancer and colon carcinoma patients was comparable or less than that in the normal controls. However, the miR-30a-5p level in the urine of patients with ovarian serous adenocarcinoma was significantly elevated compared to the healthy controls. This tissue-specific expression of miR-30a-5p makes it particularly suitable as a biomarker for ovarian serous adenocarcinoma. Recently, Jiang et al showed that miR-30a-5p was among novel non-invasive biomarkers for the diagnosis and recurrence of bladder cancer (21). Wang et al found that miR-30a-5p was upregulated in glioma samples as compared to the normal brain tissues, and its expression level was positively correlated with tumor grade of malignancy (22). Notably, we found that the urinary miR-30a-5p level was higher in stage I-II ovarian serous adenocarcinoma samples than that in stage III-IV samples, and also higher in well or moderately differentiated ovarian serous adenocarcinoma samples than in poorly differentiated samples. We hypothesized that the upregulation of miR-30a-5p may be associated with earlier stages or low-invasive features of ovarian serous adenocarcinoma. Consistent with these data, the low-invasion ovarian cell lines expressed higher levels of miR-30a-5p than the high-invasion cell lines. However, we found that the upregulated miR-30a-5p level in the urine was associated with lymphatic metastasis.

We also showed that the expression pattern of miR-30a-5p in tissues was similar to that in urine, demonstrated by a significantly higher level of miR-30a-5p in ovarian serous adenocarcinoma tissues, when compared with that in healthy controls or patients with benign gynecological diseases. Additionally, the expression of miR-30a-5p in ovarian cancer cell lines was upregulated when compared with that in normal human ovarian epithelial cells or non-ovarian cells. These results suggest that the urinary miR-30a-5p may be derived from the ovarian cancer cells within the tumor tissues. To further verify the ovarian serous adenocarcinoma origin of miR-30-5p in the urine, we compared miR-30a-5p levels in the urine of 15 ovarian serous adenocarcinoma patients before and after surgical resection of tumor tissues. The pre-operative miR-30a-5p level in the urine was significantly higher than the postoperative miR-30a-5p level, indicating that the ovarian serous adenocarcinoma is the original source of miR-30a-5p in the urine.

Exosomes are small $(30-120 \mathrm{~nm})$ membrane vesicles that may be released from different cell types including cancer cells to the urine (23). It has been demonstrated that exosomes can be isolated from various body fluids including plasma, malignant ascites, amniotic fluid, breast milk, saliva and urine $(24,25)$. Furthermore, extracellular miRNAs in exosomes are hypothesized to play a role in cellto-cell communication and to serve as biomarkers for human diseases (25). Taylor and Gercel-Taylor showed that miRNAs in exosomes from ovarian cancer patients exhibited similar profiles with miRNAs in tumor cells from the same ovarian cancer patients (19), which is in agreement with our findings that the miR-30a-5p level in exosomes and cells showed a similar expression pattern.

As the expression level of miR-30a-5p was significantly upregulated in urine and tumor tissues from ovarian serous adenocarcinoma patients, it plays an oncogenic role in ovarian cancer. To investigate the exact role of miR-30a-5p in ovarian cancer, we inhibited its expression in ovarian cancer cell lines, and showed that knockdown of miR-30a-5p significantly suppressed cell proliferation and migration of ovarian cancer cells. miR-30a-5p has been found to be deregulated in several other types of human cancers, such as glioma, colon cancer, thyroid anaplastic carcinomas and gastric cancer $(22,26-28)$. Moreover, the exact role of miR-30a-5p is tumor-specific. For instance, miR-30a-5p acts as a tumor suppressor in colon carcinoma by targeting DTL (29). Knockdown of miR-30a-5p using antisense oligonucleotide suppresses glioma cell growth by targeting SEPT7, suggesting that it acts as an oncogenic miRNA in glioma (30).

To the best of our knowledge, this is the first use of microarray technology to investigate the urine miRNA profiling of ovarian serous adenocarcinoma. The extraction of miRNA and the identification of miRNAs from the urine are feasible and practicable for the diagnosis of serous epithelial ovarian cancer. Moreover, we have demonstrated for the first time that miR-30a-5p was increased in the urine of ovarian serous adenocarcinoma patients, and inhibition of miR-30a-5p suppressed 
the malignant phenotypes of ovarian cancer in vitro. Therefore, miR-30a-5p serves as a promising diagnostic and therapeutic target for ovarian serous adenocarcinoma.

\section{Acknowledgements}

This study was financially supported by the Technology Plan of Science and Technology Bureau Hunan Province 2014FJ3090, and the National Natural Science Foundation of China nos. 81172469 and 81102009.

\section{References}

1. Siegel R, Naishadham D and Jemal A: Cancer statistics, 2012. CA Cancer J Clin 62: 10-29, 2012.

2. Wright JD, Shah M, Mathew L, Burke WM, Culhane J, Goldman N, Schiff PB and Herzog TJ: Fertility preservation in young women with epithelial ovarian cancer. Cancer 115 4118-4126, 2009

3. van Nagell JR Jr and Hoff JT: Transvaginal ultrasonography in ovarian cancer screening: Current perspectives. Int J Womens Health 6: 25-33, 2013.

4. Leung F, Diamandis EP and Kulasingam V: Ovarian cancer biomarkers: Current state and future implications from highthroughput technologies. Adv Clin Chem 66: 25-77, 2014.

5. Jacobs I and Bast RC Jr: The CA 125 tumour-associated antigen: A review of the literature. Hum Reprod 4: 1-12, 1989.

6. Cohen JG, White M, Cruz A and Farias-Eisner R: In 2014, can we do better than CA 125 in the early detection of ovarian cancer? World J Biol Chem 5: 286-300, 2014.

7. Berek JS and Bast RC Jr: Ovarian cancer screening. The use of serial complementary tumor markers to improve sensitivity and specificity for early detection. Cancer 76 (Suppl 10): S2092-S2096, 1995.

8. Ambros V: The functions of animal microRNAs. Nature 431: 350-355, 2004

9. Lewis BP, Burge CB and Bartel DP: Conserved seed pairing, often flanked by adenosines, indicates that thousands of human genes are microRNA targets. Cell 120: 15-20, 2005.

10. Calin GA and Croce CM: MicroRNA signatures in human cancers. Nat Rev Cancer 6: 857-866, 2006.

11. Bartel DP: MicroRNAs: Genomics, biogenesis, mechanism, and function. Cell 116: 281-297, 2004.

12. Lu J, Getz G, Miska EA, Alvarez-Saavedra E, Lamb J, Peck D, Sweet-Cordero A, Ebert BL, Mak RH, Ferrando AA, et al: MicroRNA expression profiles classify human cancers. Nature 435: 834-838, 2005.

13. Neal CS, Michael MZ, Pimlott LK, Yong TY, Li JY and Gleadle JM: Circulating microRNA expression is reduced in chronic kidney disease. Nephrol Dial Transplant 26: 3794-3802, 2011.

14. Lorenzen JM, Volkmann I, Fiedler J, Schmidt M, Scheffner I, Haller H, Gwinner W and Thum T: Urinary miR-210 as a mediator of acute T-cell mediated rejection in renal allograft recipients. Am J Transplant 11: 2221-2227, 2011.
15. Hanke M, Hoefig K, Merz H, Feller AC, Kausch I, Jocham D, Warnecke JM and Sczakiel G: A robust methodology to study urine microRNA as tumor marker: microRNA-126 and microRNA-182 are related to urinary bladder cancer. Urol Oncol 28: 655-661, 2010.

16. Wang G, Tam LS, Li EK, Kwan BC, Chow KM, Luk CC, Li PK and Szeto CC: Serum and urinary cell-free miR-146a and miR-155 in patients with systemic lupus erythematosus. J Rheumatol 37: 2516-2522, 2010.

17. Luo Y, Wang C, Chen X, Zhong T, Cai X, Chen S, Shi Y, Hu J, Guan X, Xia Z, et al: Increased serum and urinary microRNAs in children with idiopathic nephrotic syndrome. Clin Chem 59: 658-666, 2013

18. Iorio MV, Visone R, Di Leva G, Donati V, Petrocca F, Casalini P, Taccioli C, Volinia S, Liu CG, Alder H, et al: MicroRNA signatures in human ovarian cancer. Cancer Res 67: 8699-8707, 2007.

19. Taylor DD and Gercel-Taylor C: MicroRNA signatures of tumorderived exosomes as diagnostic biomarkers of ovarian cancer. Gynecol Oncol 110: 13-21, 2008

20. Resnick KE, Alder H, Hagan JP, Richardson DL, Croce CM and Cohn DE: The detection of differentially expressed microRNAs from the serum of ovarian cancer patients using a novel real-time PCR platform. Gynecol Oncol 112: 55-59, 2009.

21. Jiang X, Du L, Wang L, Li J, Liu Y, Zheng G, Qu A, Zhang X, Pan H, Yang Y, et al: Serum microRNA expression signatures identified from genome-wide microRNA profiling serve as novel noninvasive biomarkers for diagnosis and recurrence of bladder cancer. Int J Cancer 136: 854-862, 2015.

22. Wang K, Jia Z, Zou J, Zhang A, Wang G, Hao J, Wang Y, Yang S and Pu P: Analysis of hsa-miR-30a-5p expression in human gliomas. Pathol Oncol Res 19: 405-411, 2013.

23. Vlassov AV, Magdaleno S, Setterquist R and Conrad R: Exosomes: Current knowledge of their composition, biological functions, and diagnostic and therapeutic potentials. Biochim Biophys Acta 1820: 940-948, 2012.

24. Gu Y, Li M, Wang T, Liang Y, Zhong Z, Wang X, Zhou Q, Chen L, Lang Q,He Z, et al: Lactation-related microRNA expression profiles of porcine breast milk exosomes. PLoS One 7: e43691, 2012.

25. Keller S, Ridinger J, Rupp AK, Janssen JW and Altevogt P: Body fluid derived exosomes as a novel template for clinical diagnostics. J Transl Med 9: 86, 2011.

26. Xi Y, Formentini A, Chien M, Weir DB, Russo JJ, Ju J, Kornmann $\mathrm{M}$ and $\mathrm{Ju} \mathrm{J}$ : Prognostic values of microRNAs in colorectal cancer. Biomark Insights 2: 113-121, 2006.

27. Visone R, Pallante P, Vecchione A, Cirombella R, Ferracin M, Ferraro A, Volinia S, Coluzzi S, Leone V, Borbone E, et al: Specific microRNAs are downregulated in human thyroid anaplastic carcinomas. Oncogene 26: 7590-7595, 2007.

28. Li X, Zhang Y, Zhang Y, Ding J, Wu K and Fan D: Survival prediction of gastric cancer by a seven-microRNA signature. Gut 59: 579-585, 2010.

29. Baraniskin A, Birkenkamp-Demtroder K, Maghnouj A, Zöllner H, Munding J, Klein-Scory S, Reinacher-Schick A, Schwarte-Waldhoff I, Schmiegel W and Hahn SA: MiR-30a-5p suppresses tumor growth in colon carcinoma by targeting DTL. Carcinogenesis 33: 732-739, 2012.

30. Jia Z, Wang K, Wang G, Zhang A and Pu P: MiR-30a-5p antisense oligonucleotide suppresses glioma cell growth by targeting SEPT7. PLoS One 8: e55008, 2013. 